\title{
UM RETRATO DA PRIMEIRA INFÂNCIA EM SITUAÇÃO DE POBREZA
}

\section{A portrait of first childhood in poverty \\ Un retrato de la primera infancia en situación de pobreza}

\author{
Raissa Freitas Gomes Brito \\ Universidade de Fortaleza - UNIFOR - Fortaleza (CE) - Brasil
}

Herika Paiva Pontes

Universidade de Fortaleza - UNIFOR - Fortaleza (CE) - Brasil

Mirna Albuquerque Frota

Universidade de Fortaleza - UNIFOR - Fortaleza (CE) - Brasil

\section{RESUMO}

Objetivo: Conhecer o perfil da primeira infância em situação de pobreza no estado do Ceará. Métodos: Trata-se de estudo descritivo, de natureza quantitativa, realizado com crianças de zero a seis anos em situação de pobreza no estado do Ceará. Os dados foram coletados na plataforma Tabulador de Informações do Cadastro Único do Ministério do Desenvolvimento Social, no período de junho de 2017, quando o mesmo contava com 5.385 .085 pessoas cadastradas, das quais 581.336 tinham de zero a seis anos, definindo a amostra da pesquisa. As variáveis coletadas foram: sexo, etnia, renda, habitação, educação, saúde e violação dos direitos, para serem analisadas descritivamente. Resultados: Com relação à renda, são 552.921 famílias (95,11\%) consideradas de baixa renda, das quais 73,18\% (425.441) vivem em situação de extrema pobreza. Apesar de a maioria (388.325; $66,79 \%)$ morar em domicílios situados em zonas urbanas, em casas predominantemente de alvenaria $(548.871 ; 94,41 \%)$, $281.726(41,02 \%)$ residem em domicílio sem nenhum tipo de calçamento em frente à residência e $427.906(63,31)$, sem rede coletora de esgoto. Na dimensão educação, 395.776 crianças $(68,08 \%)$ não frequentam a escola e menos de $1 \%(70)$ vive em situação de rua e trabalho infantil. Foram, predominante, crianças do sexo masculino $(297.786 ; 51,22 \%)$ e consideradas, por seus responsáveis, pardas (479.769; 82,53\%). Conclusão: A maioria das crianças de até 6 anos e em situação de pobreza no Ceará são do sexo masculino, de cor parda, residindo em domicílios com precárias condições de saneamento básico e ainda sem acesso à educação infantil.

Descritores: Criança; Pobreza; Desenvolvimento Infantil.

\section{ABSTRACT}

Objective: To know the profile of early childhood in poverty in the state of Ceará. Methods: This quantitative descriptive study was carried out with children aged zero to six years living in poverty in the state of Ceará. Data were collected from the Information Tabulator platform of the Unified Registration system of the Ministry of Social Development in June 2017, when there were 5,385,085 people registered. Of these, 581,336 were between zero and six years old and were included in the research sample. Information on sex, ethnicity, income, housing, education, health and violation of human rights were descriptively analyzed. Results: With regard to income, 552,921 families (95.11\%) were low-income, $73.18 \%(425,441)$ of whom lived in extreme poverty. Although the majority $(388,325 ; 66.79 \%)$ lived in houses located in urban areas and in predominantly masonry houses (548,871; 94.41\%), 281,726 (41.02\%) children lived in houses without any type of pavement in front of the house and 427,906 (63.31) did not have access to sewage collection network. As for education, 395,776 children (68.08\%) did not go to school and less than $1 \%$ (70) lived in the street and were submitted to child labor. There was a predominance of male children (297,786; 51.22\%) and children considered Pardas (mixed-race Brazilians) (479,769; 82.53\%) by their parents. Conclusion: The majority of children up to the age of six who lived in poverty in Ceará were male, Pardas, and lived houses with poor access to basic sanitation and without access to early childhood education.

Descriptors: Child; Poverty; Child Development. 


\section{RESUMEN}

Objetivo: Conocer el perfil de la primera infancia en situación de pobreza en el estado de Ceará. Métodos: Se trata de un estudio descriptivo, de naturaleza cuantitativa realizado con niños entre cero y seis años en situación de pobreza en el estado de Ceará. Se recogieron los datos en la plataforma Tabulador de Informaciones del Registro Único del Ministerio del Desarrollo Social en el período de junio de 2017 cuando el mismo tenía 5.385.085 personas registradas de las cuales 581.336 tenían entre cero y seis años lo que definió la muestra de la investigación. Las variables recogidas fueron el sexo, la etnia, la renta, la vivienda, la educación, la salud y la violación de los derechos y todas fueron analizadas de modo descriptivo. Resultados: Respecto la renta, 552.921 familias (95,11\%) son consideradas de baja renta de las cuales el 73,18\% (425.441) viven en situación de extrema pobreza. Aunque la mayoría (388.325; 66,79\%) vive en domicilios de las zonas urbanas y la mayoría en casas de albañilería (548.871; 94,41\%), 281.726 (41,02\%) viven en domicilio sin ningún tipo de pavimentación delante de su vivienda y 427.906 $(63,31)$ no tiene red colectora de aguas residuales. En la dimensión educación, 395.776 niños $(68,08 \%)$ no frecuentan la escuela y menos del $1 \%$ (70) vive en la calle y trabajan. Ha predominado niños del sexo masculino (297.786; 51,22\%) y considerados pardos por sus responsables (479.769; 82,53\%). Conclusión: La mayoría de los niños de hasta 6 años y en situación de pobreza en Ceará son del sexo masculino, de color pardo, viviendo en domicilios con precarias condiciones de saneamiento básico $y$ todavía sin acceso a la educación infantil.

Descriptores: Niño; Pobreza; Desarrollo Infantil.

\section{INTRODUÇÃO}

A saúde das crianças é um dos assuntos mais importantes do mundo atualmente. A Organização das Nações Unidas e alguns de seus organismos vêm convocando os países a construírem "um mundo para as crianças". No Brasil, a Constituição Federal atribui prioridade absoluta aos direitos da criança e do adolescente, não podendo ser, portanto, nenhum outro assunto ou demanda, mais importante do que as medidas que garantam o cumprimento dos seus direitos ${ }^{(1)}$.

De acordo com o último Censo Demográfico, das crianças existentes no Brasil em 2010, 19.632.111 tinham até seis anos de idade, o que correspondia a mais de $10 \%$ da população. Ao se comparar com outros países, esse contingente populacional impõe um desafio às políticas públicas. Por exemplo, o Brasil possuía, à época, mais indivíduos nessa faixa etária do que a população inteira do Chile e ainda o equivalente a duas vezes a população total da Suécia(2).

Esse período que compreende os seis primeiros anos de vida, denominado de primeira infância, é uma etapa de extrema importância, durante a qual a criança passa por significativos processos de desenvolvimento, que são influenciados pela realidade e o contexto nos quais está inserida. Entre esses processos, estão: o crescimento físico, o amadurecimento cerebral, a aquisição dos movimentos, o desenvolvimento da capacidade de aprendizado e a iniciação social e afetiva ${ }^{(3)}$.

Prejuízos no desenvolvimento das crianças nesse período (como as deficiências nutricionais, por exemplo) terão, portanto, profundos impactos para o resto da vida do indivíduo e, nesse cenário, destacam-se as implicações da pobreza e da desigualdade social para as condições de saúde da população. Um estudo recente com mais de um milhão de pessoas evidenciou, inclusive, que a pobreza encurta a vida muito mais do que a obesidade, a hipertensão e o consumo excessivo de álcool(4).

A íntima relação entre saúde e as condições de vida torna-se notória ao se observar que os indivíduos mais afetados nas epidemias que ocorreram durante o século $\mathrm{XX}$, por exemplo, eram os que, em geral, viviam na periferia, em moradias pouco espaçosas, sem estrutura de saneamento básico, com alimentação inadequada e renda insuficiente para manter condições mínimas de sobrevivência ${ }^{(5)}$.

Todavia, apesar da relevância do problema, a pobreza, assim como a sua relação com as condições de saúde, continua sendo um tema negligenciado em grande parte do mundo. Uma prova disso é que, em 2011, os estados membros da OMS, ao assinarem um plano para reduzir a mortalidade devido a doenças transmissíveis em $25 \%$ até 2025 , não incluíram os fatores socioeconômicos no plano(6).

Diante desse contexto, a realização de um diagnóstico das reais condições de vida na primeira infância é o primeiro e mais importante passo a ser dado a fim de nortear o planejamento de ações que venham a efetivamente suprir as necessidades dessas crianças, cumprindo, assim, a determinação do Marco Legal da Primeira Infância de que as políticas públicas voltadas ao atendimento dos direitos das crianças de zero a seis anos sejam elaboradas 
e executadas com vistas ao atendimento integral; estabelecendo, ainda, a priorização do atendimento daquelas famílias em situação de vulnerabilidade ou com direitos violados, bem como as que têm crianças com indicadores de risco ou deficiência ${ }^{(7)}$.

A partir disso, em decorrência da evidência de que, dentre outros fatores, a pobreza na primeira infância pode ser uma barreira trágica ao desenvolvimento integral da criança, a pesquisa desenvolvida teve como objetivo conhecer o perfil da primeira infância em situação de pobreza no estado do Ceará.

\section{MÉTODOS}

Trata-se de uma pesquisa transversal, com abordagem quantitativa e descritiva(8), realizada em junho de 2017.

Seguindo um critério legal estabelecido pelo Marco Legal da Primeira Infância, que preconiza a priorização do atendimento às famílias em situação de vulnerabilidade( ${ }^{(6)}$, a pesquisa teve como população-alvo as crianças de zero a seis anos em situação de pobreza no estado do Ceará. Destaca-se, contudo, que, segundo o Ministério do Desenvolvimento Social (MDS), ao falar-se de famílias em situação de pobreza, está-se referindo àquelas com renda per capita de até 170,00 reais mensais ${ }^{(8)}$.

A coleta de dados ocorreu por meio do Cadastro Único para Programas Sociais do Governo Federal (Cadastro Único), que é um instrumento que identifica e caracteriza as famílias de baixa renda no Brasil, permitindo que o governo conheça melhor sua realidade socioeconômica por meio do registro de informações como características habitacionais, composição familiar, escolaridade, situação de renda, entre outras; funcionando como uma porta de entrada para as famílias acessarem diversas políticas públicas.

Os dados coletados, disponíveis na plataforma Tabulador de Informações do Cadastro Único (TabCad) do MDS, fazem referência ao período de junho de 2017, quando o Cadastro Único contava com 5.385 .085 pessoas cadastradas, das quais 10,8\% (581.336) tinham de zero a seis anos, vivenciando, portanto, a primeira infância, e foram essas crianças que compuseram a amostra da pesquisa.

As variáveis investigadas foram categorizadas em seis dimensões e codificadas conforme descrito no Quadro 1. Por fim, realizou-se, através de cálculos estatísticos de frequência simples, a análise descritiva dos dados coletados.

Os dados utilizados no estudo são de domínio público e, em decorrência disso, não houve necessidade de aprovação por Comitê de Ética em Pesquisa.

Quadro I - Categorização das dimensões e variáveis investigadas retiradas da plataforma tabulador de informações do cadastro único do Ministério do Desenvolvimento Social referente a junho de 2017.

\begin{tabular}{|ll|}
\hline Dimensão & Variável \\
\hline \multirow{2}{*}{ Renda (R) } & R1. Faixa de renda total da família \\
& R2. Faixa de renda per capita da família \\
& R3. Beneficiária do Programa Bolsa-Família \\
& H1. Calçamento em frente ao domicílio \\
& H2. Zona do domicílio \\
& H3. Existência de banheiro \\
& H4. Forma de abastecimento de água \\
& H5. Forma de coleta de lixo \\
Habitação (H) & H6. Forma de escoamento sanitário \\
& H7. Material predominante nas paredes externas do domicílio \\
& H8. Material predominante no piso do domicílio \\
& H1. Existência de água canalizada no domicílio \\
& H10. Tipo de iluminação presente no domicílio \\
Educação (E) & E1. Inserção na escola \\
Saúde (S) & S1. Pessoa com deficiência \\
Violação de Direitos (VD) & VD1. Situação de rua \\
& VD2. Situação de trabalho infantil \\
Outros (O) & O1. Sexo \\
& O2. Cor ou Raça \\
\hline
\end{tabular}




\section{RESULTADOS}

Das 581.336 crianças de zero a seis anos que compuseram a amostra, $372.774(64,12 \%)$ encontram-se na faixa de zero a quatro anos. No que se refere à dimensão renda (Tabela I), $552.921(95,11 \%)$ pertencem a famílias consideradas de baixa renda, que contam com até 1 salário mínimo mensal ( $R \$ 937,00$, à época) para suprir suas necessidades básicas. Dessas, $425.441(73,18 \%)$ pertencem a famílias com renda per capita de até $R \$ 85,00$, consideradas, pelo governo federal, em situação de extrema pobreza. Outras $(76.266 ; 13,12 \%)$, com renda entre $\mathrm{R} \$ 85,01$ e $\mathrm{R} \$ 170,00$.

No que diz respeito às condições habitacionais (Tabela II), apesar de a maioria $(388.325 ; 66,79 \%)$ morar em domicílios situados em zonas urbanas e em casas de alvenaria $(548.871 ; 94,41 \%)$, uma quantidade considerável de crianças $(281.726 ; 41,02 \%)$ reside em domicílio sem nenhum tipo de calçamento em frente e sem banheiro (56.503; 9,72\%). Além disso, 427.906 (63,31\%), uma maioria significativa da amostra, reside em domicílios sem rede coletora de esgoto, ou seja, em áreas sem saneamento básico.

Quanto ao lixo, $426.376(73,34 \%)$ das residências o têm coletado, mesmo que 38.100 (6,55\%) de forma indireta. Contudo, ainda são $151.621(26,07 \%)$ crianças em moradias que não têm o lixo coletado. Além disso, também chama atenção o fato de que 26.581 (4,57\%) moram em domicílios revestidos com material precário, como taipa e madeira aproveitada.

Tabela I - Distribuição absoluta e relativa das categorias da dimensão renda das famílias de crianças de zero a seis anos em situação de pobreza no estado do Ceará. 2017.

\begin{tabular}{lccc}
\hline Variável & Resposta & $\mathbf{n}$ & $\%$ \\
\hline Dimensão Renda (R) & & & \\
& Até 1 & 552.921 & 95,11 \\
R1. Faixa de renda total da família (salário mínimo)* & Entre 1 e 2 & 25.213 & 4,34 \\
& Entre 2 e 3 & 2.684 & 0,46 \\
& Acima de 3 & 518 & 0,09 \\
& Sem resposta & 0 & 0 \\
& Até 85,00 & 425.441 & 73,18 \\
R2. Faixa de renda per capita da família (reais) & 85,01 a 170,00 & 76.266 & 13,12 \\
& 170,01 a 468,00 & 74.640 & 12,83 \\
& Acima de 468,00 & 4.989 & 0,86 \\
R3. Beneficiária do Programa Bolsa Família & Sem resposta & 0 & 0 \\
& Não & 124.635 & 21,44 \\
& Sim & 456.701 & 78,56 \\
\hline
\end{tabular}

* Valor referente ao salário mínimo de R\$: 937,00

Na dimensão educação (Tabela III), verificou-se que existem pelo menos $395.776(68,08 \%)$ crianças que ainda não frequentam a creche ou escola. Dentre as que já o fazem, 179.806 (30,93\%), a maioria é usuária da rede pública de ensino, como esperado, tendo em vista que se tratam de indivíduos em condições de baixa renda. Com relação à saúde, constatou-se que $4.411(0,76 \%)$ crianças da amostra foram cadastradas como tendo algum tipo de deficiência. Entretanto, não foi possível identificar quais os tipos de deficiência mais recorrentes, porque aproximadamente $99 \%$ dos cadastros não registraram essa informação.

No que tange às "Violações de direitos" sofridas pela amostra, o sistema só registra aquelas relacionadas à situação de rua e trabalho infantil. Ambas apresentaram uma prevalência que não chega a representar $1 \%$ da amostra, com 53 crianças vivendo na rua e 17 realizando trabalho infantil. Salienta-se, contudo, que, ao tratar-se dessas questões, o objetivo é sempre alcançar o indicador zero.

Por fim, percebeu-se, na dimensão "Outros" que, no que concerne ao sexo, há um equilíbrio entre meninos e meninas, com uma pequena prevalência do sexo masculino, que superou em 2,44\% (14.236) o feminino. Quanto à cor, $479.769(82,53 \%)$ das crianças foram declaradas por seus responsáveis como pardas. 
Tabela II - Distribuição das categorias da dimensão habitação de crianças de zero a seis anos em situação de pobreza no estado do Ceará. 2017.

\begin{tabular}{|c|c|c|c|}
\hline Variável & Resposta & $\mathbf{n}$ & $\%$ \\
\hline \multicolumn{4}{|l|}{ Dimensão Habitação (H) } \\
\hline \multirow{4}{*}{ H1. Calçamento em frente ao domicílio } & Total & 281.726 & 48,46 \\
\hline & Parcial & 57.803 & 9,94 \\
\hline & Não existe & 238.466 & 41,02 \\
\hline & Sem resposta & 3.341 & 0,57 \\
\hline \multirow{3}{*}{ H2. Zona do domicílio } & Urbana & 388.325 & 66,79 \\
\hline & Rural & 192.958 & 33,19 \\
\hline & Sem resposta & 53 & 0,01 \\
\hline \multirow{3}{*}{ H3. Existência de banheiro } & Sim & 521.494 & 89,71 \\
\hline & Não & 56.503 & 9,72 \\
\hline & Sem resposta & 3.339 & 0,57 \\
\hline \multirow{5}{*}{ H4. Forma de abastecimento de água } & Rede geral de distribuição & 403.600 & 69,43 \\
\hline & Poço ou nascente & 109.023 & 18,75 \\
\hline & Cisterna & 26.670 & 4,59 \\
\hline & Outra forma & 38.704 & 6,66 \\
\hline & Sem resposta & 3.339 & 0,57 \\
\hline \multirow{7}{*}{ H5. Forma de coleta do lixo } & Coletado diretamente & 388.276 & 66,79 \\
\hline & Coletado indiretamente & 38.100 & 6,55 \\
\hline & Queimado ou enterrado na propriedade & 137.365 & 23,62 \\
\hline & Jogado em terreno baldio ou logradouro & 12.901 & 2,21 \\
\hline & Jogado em rio ou mar & 326 & 0,06 \\
\hline & Tem outro destino & 1.029 & 0,18 \\
\hline & Sem resposta & 3.339 & 0,57 \\
\hline \multirow{7}{*}{ H6. Forma de escoamento sanitário } & Rede coletora de esgoto ou pluvial & 153.430 & 26,39 \\
\hline & Fossa séptica & 126.136 & 21,70 \\
\hline & Fossa rudimentar & 229.649 & 39,50 \\
\hline & Vala a céu aberto & 5.742 & 0,99 \\
\hline & Direto para um rio, lago ou mar & 1.519 & 0,26 \\
\hline & Outra forma & 5.018 & 0,86 \\
\hline & Sem resposta & 59.842 & 10,29 \\
\hline \multirow{9}{*}{$\begin{array}{l}\text { H7. Material predominante nas paredes externas } \\
\text { do domicílio }\end{array}$} & Alvenaria/tijolo com revestimento & 446.017 & 76,72 \\
\hline & Alvenaria/tijolo sem revestimento & 102.854 & 17,69 \\
\hline & Madeira aparelhada & 264 & 0,05 \\
\hline & Taipa revestida & 14.694 & 2,53 \\
\hline & Taipa não revestida & 11.887 & 2,04 \\
\hline & Madeira aproveitada & 596 & 0,10 \\
\hline & Palha & 141 & 0,02 \\
\hline & Outro material & 1.453 & 0,25 \\
\hline & Sem resposta & 3.339 & 0,57 \\
\hline \multirow{8}{*}{ H8. Material predominante no piso do domicílio } & Terra & 21.060 & 3,62 \\
\hline & Cimento & 412.332 & 70,92 \\
\hline & Madeira aproveitada & 385 & 0,07 \\
\hline & Madeira aparelhada & 330 & 0,06 \\
\hline & Cerâmica, lajota ou pedra & 139.309 & 23,96 \\
\hline & Carpete & 236 & 0,04 \\
\hline & Outro material & 4.345 & 0,75 \\
\hline & Sem resposta & 3.339 & 0,57 \\
\hline \multirow{3}{*}{ H9. Existência de água canalizada no domicílio } & Sim & 460.500 & 79,21 \\
\hline & Não & 117.497 & 20,21 \\
\hline & Sem resposta & 3.339 & 0,57 \\
\hline \multirow{7}{*}{ H10. Tipo de iluminação presente no domicílio } & Elétrica com medidor próprio & 529.291 & 91,04 \\
\hline & Elétrica com medidor comunitário & 8.071 & 1,39 \\
\hline & Elétrica sem medidor & 21.873 & 3,76 \\
\hline & Óleo, querosene ou gás & 3.062 & 0,53 \\
\hline & Vela & 2.243 & 0,39 \\
\hline & Outra forma & 13.457 & 2,31 \\
\hline & Sem resposta & 3.339 & 0,57 \\
\hline
\end{tabular}


Tabela III - Distribuição absoluta e relativa das categorias das dimensões educação, saúde, violação de direitos e outros de crianças de zero a seis anos em situação de pobreza no estado do Ceará. 2017.

\begin{tabular}{llcc}
\hline Variável & Resposta & $\mathbf{n}$ & $\%$ \\
\hline Dimensão Educação (E) & Sim, rede pública & 162.360 & 27,93 \\
& Sim, rede particular & 17.446 & 3,00 \\
E1. Inserção na escola & Não, já frequentou & 842 & 0,14 \\
& Nunca frequentou & 394.934 & 67,94 \\
& Sem resposta & 5.754 & 0,99 \\
Dimensão Saúde (S) & & & \\
& Sim & 4.411 & 0,76 \\
S1. Pessoa com Deficiência & Não & 576.925 & 99,24 \\
& Sem resposta & 0 & 0 \\
Dimensão Violação de Direitos (VD) & Sim & 53 & 0,01 \\
& Não & 581.283 & 99,99 \\
VD1. Situação de rua & Sem resposta & 0 & 0 \\
& Sim & 17 & 0,003 \\
VD2. Situação de trabalho infantil & Não & 581.319 & 99,997 \\
Dimensão Outros (O) & Sem resposta & 0 & 0 \\
O1. Sexo & Masculino & 297.786 & 51,22 \\
& Feminino & 283.550 & 48,78 \\
& Sem resposta & 0 & 0 \\
& Branca & 95.038 & 16,35 \\
O2. Cor ou Raça & Preta & 2.705 & 0,47 \\
& Amarela & 2.529 & 0,44 \\
& Parda & 479.769 & 82,53 \\
& Indígena & 1.295 & 0,22 \\
\hline & Sem resposta & 0 & 0 \\
\hline
\end{tabular}

\section{DISCUSSÃO}

A fase denominada de primeiríssima infância é caracterizada pela faixa etária que vai de zero a três anos, período decisivo para a estruturação física e psíquica do indivíduo. Vale ressaltar que esses aspectos estão interligados e influenciam um ao outro durante toda a vida ${ }^{(10)}$.

Os dados coletados na presente pesquisa mostraram que a maior parte das crianças em situação de pobreza no estado do Ceará, inseridas no Cadastro Único (64,12\%), encontram-se nessa fase. Evidência preocupante, tendo em vista que a pobreza é considerada um relevante fator de risco para o desenvolvimento infantil, afetando negativamente as condições de saúde, educação e o alcance de oportunidades.

Estudos mostram a associação entre a pobreza extrema e atrasos no desenvolvimento de crianças. Diante disso, faz-se necessário conhecer, a fundo, as condições de vida dessas crianças, para que se possa planejar políticas públicas voltadas ao seu desenvolvimento integral ${ }^{(11)}$.

Outro dado que chamou atenção foi o de que ainda são, pelo menos, 427.906 crianças, no estado do Ceará, residindo em domicílios sem rede coletora de esgoto, ou seja, em áreas sem saneamento básico, apesar de ser um direito essencial garantido constitucionalmente no Brasil havendo um reflexo das profundas implicações desse serviço para a saúde pública e ambiental, visto que sua carência pode afetar negativamente em campos essenciais, como educação, trabalho, economia, biodiversidade, disponibilidade hídrica, entre outras ${ }^{(12)}$.

Tal indicador, quando analisado juntamente com os outros evidenciados neste estudo, apontam para um grave quadro social de pobreza que ainda permeia o Ceará, a despeito de propagandas eleitorais recorrentes no Brasil, que publicizam que a pobreza vem sendo eliminada no país.

Fato também considerável no estudo é que a maioria das crianças em situação de pobreza não frequentam a escola, contrariando o Estatuto da Criança e do Adolescente, que diz ser um direito que deve ser garantido a todas 
as crianças, sendo dever do Estado assegurar, àquelas de zero a cinco anos de idade, o atendimento em creche ou pré-escola ${ }^{(13)}$.

Diante do exposto, as condições de vida das crianças estudadas se mostraram extremamente desfavoráveis, com pouco acesso à saúde, educação, habitação, bens e serviços públicos. Esses dados contribuem para auxiliar no planejamento de políticas públicas que visem à mudança desse cenário, tendo em vista a garantia dos direitos das crianças estabelecidos pela Constituição Federal e a obtenção de perspectivas futuras mais positivas. Salienta-se, ainda, a necessidade de que os brasileiros fortaleçam seu compromisso com a cidadania, cobrando das autoridades/ governantes a adoção de medidas mais enérgicas e eficazes de combate à pobreza no país. Não se justifica um país que tem uma das mais elevadas cargas tributárias ter uma alta taxa de pobreza.

As limitações do presente estudo denotam-se no fato dos dados retratarem um único mês, além de terem sido coletados de um banco secundário, que apresenta campos sem preenchimento, influenciando nos resultados. Sugere-se que estudos posteriores analisem de forma mais profunda cada dimensão estudada e sua relação com desenvolvimento infantil durante a primeira infância.

\section{CONCLUSÃO}

A maioria das crianças de até seis anos de idade, em situação de pobreza, cadastradas no Cadastro Único do Governo Federal, são do sexo masculino, de cor parda, residindo em áreas urbanas, em domicílios com precárias condições de saneamento básico e ainda sem acesso à educação infantil.

\section{CONFLITOS DE INTERESSE}

Os autores declaram não haver conflitos de interesse.

\section{REFERÊNCIAS}

1. Laffite LTG, Didonet V. A intersetorialidade nas políticas para a primeira infância [Internet]. Rio de Janeiro: Rede Nacional Primeira Infância; 2015. [acesso em 2017 Ago 20]. Disponível em: https://undime.org.br/uploads/ documentos/phpcrSG7h_55a64dbb2efb2.pdf

2. Chiesa AM, Sousa FLP, Cypel S, Oliveira ZMR. Primeiríssima infância da gestação aos três anos: percepções e práticas da sociedade brasileira sobre a fase inicial da vida. São Paulo: Fundação Maria Cecília Souto Vidigal; 2013.

3. Eyken EDV, Ribeiro CDM. Desenvolvimento infantil: seus agentes e as políticas públicas do município do Rio de Janeiro. Physis. 2012;22(3):1085-99.

4. Stringhini S, Carmeli C, Jokela M, Avendaño M. Muennig P, Guida F, et al. Socioeconomic status and the $25 \times 25$ risk factors as determinants of premature mortality: a multicohort study and meta-analysis of 1.7 million men and women. Lancet [Internet]. 2017 [acesso em 2018 Set 05];389(10075):1229-37. Disponível em: https://www. thelancet.com/journals/lancet/article/PIIS0140-6736(16)32380-7/fulltext?code=lancet-site

5. Cavalcante LIC, Araújo CR, Góes ELC, Magalhães CMC. Análise das condições sociofamiliares de crianças em acolhimento institucional: comparando dois momentos. Psicol Argum. 2014;32(76):79-92.

6. United Nations. Resolution adopted by the General Assembly: political declaration of the high-level meeting of the General Assembly on the Prevention and Control of Non-communicable Diseases [Internet]. New York: UN; 2011 [acesso em 2014 Nov 3]. Disponível em: http://www.who.int/nmh/events/un_ncd_summit2011/political_ declaration_en.pdf?ua=

7. Brasil. Lei $n^{0} 13.257$, de 08 de Março de 2016. Dispõe sobre as políticas públicas para a primeira infância e altera o Estatuto da Criança e do Adolescente, o Código de Processo Civil, a CLT, a Lei no 11.770, de 9 de Setembro de 2008, e a Lei n 12.662, de 5 de Junho de 2012. Diário Oficial da União; Brasília, 09 Mar. 2016; Seção 1.

8. Perovano DG. Manual de metodologia científica para a segurança pública e defesa social. Curitiba: Juruá; 2014.

9. Ministério do Desenvolvimento Social (BR). Bolsa família: como funciona [Internet]. 2015 [acesso em $2015 \mathrm{Jul}$ 01]. Disponível em: http://mds.gov.br/assuntos/bolsa-familia/o-que-e/como-funciona/como-funciona]. 
10. Morais RLS, Carvalho AM, Magalhães LC, Pinto PAF. Primeira infância e pobreza no Brasil: uma análise integrada a partir de indicadores em saúde, educação e desenvolvimento social. Rev Polít Públicas. 2015;19(1):303-14.

11. Teixeira AN, Lôbo KRG, Duarte ATC. A Criança e o ambiente social: aspectos intervenientes no processo de desenvolvimento na primeira infância. Id on Line Rev Psic. 2016;10(31):114-34.

12. Hamadani JD, Tofail F, Huda SN, Alam DS, Ridout DA, Attanasio O, et al. Cognitive deficit and poverty in the first 5 years of childhood in Bangladesh. Pediatrics. 2014;134(4):1001-8.

13. Brasil. Lei n 8.069, de 13 de Julho de 1990. Dispõe sobre o Estatuto da Criança e do Adolescente e dá outras providências. Diário Oficial da União; Brasília, 13 Jul 1990; Seção 1.

\section{Endereço para correspondência:}

Raissa Freitas Gomes Brito

Universidade de Fortaleza - UNIFOR

Programa de Pós-Graduação em Saúde Coletiva

Av. Washington Soares, 1321, Bloco S, Sala 1

Bairro: Edson Queiroz

CEP: 60.811-905 - Fortaleza - CE - Brasil

E-mail: raissafreitasgomes@hotmail.com 